\title{
SOlutions OF THE DisPERSIONLESS Toda Hierarchy Constrained by String Equations *
}

\author{
Luis Martínez Alonso ${ }^{1, \ddagger}$ and Elena Medina ${ }^{2,-4}$ \\ ${ }^{1}$ Departamento de Física Teórica II, Universidad Complutense \\ E28040 Madrid, Spain \\ ${ }^{2}$ Departamento de Matemáticas, Universidad de Cádiz \\ E11510, Puerto Real, Cádiz, Spain \\ ${ }^{\ddagger}$ luism@fis.ucm.es \\ *lena.medina@uca.es
}

\begin{abstract}
Solutions of the Riemann-Hilbert problem implementing the twistorial structure of the dispersionless Toda (dToda) hierarchy are obtained. Two types of string equations are considered which characterize solutions arising in hodograph sectors and integrable structures of two-dimensional quantum gravity and Laplacian growth problems.
\end{abstract}

Key words: Dispersionless Toda hierarchy, string equations, hodograph relations.

1991 MSC: 58B20.

\footnotetext{
*Partially supported by DGCYT project BFM2002-01607
} 


\section{Introduction}

The dispersionless Toda (dToda) hierarchy [1]-3] describes several relevant integrable structures as the genus zero-limit of the Landau-Ginzburg formulation of two-dimensional string theory [4]-[6], the dynamics of conformal maps and the Laplacian growth problem governing interface dynamics [7]-8]. It can be formulated in terms of two Laurent series

$$
\mathcal{L}=p+\sum_{n \geq 0} \frac{u_{n+1}(t, \bar{t}, s)}{p^{n}}, \quad \overline{\mathcal{L}}^{-1}=\frac{\bar{u}_{0}(t, \bar{t}, s)}{p}+\sum_{n \geq 0} \bar{u}_{n+1}(t, \bar{t}, s) p^{n},
$$

with coefficients depending on the variables $t:=\left(x:=t_{1}, t_{2}, \ldots\right), \bar{t}:=(y:=$ $\left.\bar{t}_{1}, \bar{t}_{2}, \ldots\right)$ and the spacial variable $s$, and such that the following Lax equations are satisfied

$$
\begin{cases}\frac{\partial \mathcal{L}}{\partial t_{n}}=\left\{\mathcal{B}_{n}, \mathcal{L}\right\}, & \frac{\partial \overline{\mathcal{L}}}{\partial t_{n}}=\left\{\mathcal{B}_{n}, \overline{\mathcal{L}}\right\} \\ \frac{\partial \mathcal{L}}{\partial \bar{t}_{n}}=\left\{\overline{\mathcal{B}}_{n}, \mathcal{L}\right\}, & \frac{\partial \overline{\mathcal{L}}}{\partial \bar{t}_{n}}=\left\{\overline{\mathcal{B}}_{n}, \overline{\mathcal{L}}\right\}\end{cases}
$$

where the Poisson bracket is defined as

$$
\{F, G\}:=p\left(\frac{\partial F}{\partial p} \frac{\partial G}{\partial s}-\frac{\partial F}{\partial s} \frac{\partial G}{\partial p}\right) .
$$

and

$$
\mathcal{B}_{n}:=\left(\mathcal{L}^{n}\right)_{\geq 0}, \quad \overline{\mathcal{B}}_{n}:=\left(\overline{\mathcal{L}}^{-n}\right)_{\leq-1} .
$$

Here $(\ldots)_{\geq 0}\left((\ldots)_{\leq-1}\right)$ means the part of the Laurent series with positive (strictly negative) powers of $p$

The main integrable model included in the dToda hierarchy is the BoyerFinley equation 9]

$$
\frac{\partial^{2} U}{\partial x \partial y}+\frac{\partial^{2}}{\partial s^{2}}(\exp U)=0
$$

where $U=U(x, y, s):=\ln \bar{u}_{0}$. It is a much studied $(2+1)$-dimensional integrable system which appears in the classification of self-dual Einstein spaces with rotational Killing symmetry [9]-11] and in the twistor analysis of Einstein-Weyl spaces [12, 13]. 
Several methods for finding solutions of the members of the dToda hierarchy have been proposed. A general strategy within the framework of dispersionless integrable hirarchies is the hodograph method [14-[17]. Another approach, used for generating solutions of the Boyer-Finley equation, is the group foliation method of [18, 19. In the present work we are concerned with a third scheme: the twistor method of Takasaki-Takebe [1, 20]. It involves an extended Lax formalism with a pair of Orlov functions $\mathcal{M}$ and $\overline{\mathcal{M}}$ such that

$$
\{\mathcal{L}, \mathcal{M}\}=\mathcal{L}, \quad\{\overline{\mathcal{L}}, \overline{\mathcal{M}}\}=\overline{\mathcal{L}}
$$

with Laurent expansions

$$
\begin{aligned}
& \mathcal{M}=\sum_{n \geq 1} n t_{n} \mathcal{L}^{n}+s+\sum_{n \geq 1} \frac{v_{n}(t, \bar{t}, s)}{\mathcal{L}^{n}}, \\
& \overline{\mathcal{M}}=-\sum_{n \geq 1} n \bar{t}_{n} \overline{\mathcal{L}}^{-n}+s+\sum_{n \geq 1} \bar{v}_{n}(t, \bar{t}, s) \overline{\mathcal{L}}^{n},
\end{aligned}
$$

and verifying the same Lax equations (2) as $\mathcal{L}$ and $\overline{\mathcal{L}}$. Solutions of the dToda hierarchy are characterized by imposing two constraints (string equations) of the form

$$
P(\mathcal{L}, \mathcal{M})=\bar{P}(\overline{\mathcal{L}}, \overline{\mathcal{M}}), \quad X(\mathcal{L}, \mathcal{M})=\bar{X}(\overline{\mathcal{L}}, \overline{\mathcal{M}})
$$

where $(P(p, s), X(p, s))$ and $(\bar{P}(p, s), \bar{X}(p, s))$ are pairs of canonically conjugate variables (i.e. $\{P, X\}=P,\{\bar{P}, \bar{X}\}=\bar{P}$ ), which together with the conditions (11) and (7) constitute a Riemann-Hilbert problem.

In the present work we solve two types of systems of string equations and obtain solutions of the truncated $\left(n_{1}, n_{2}\right)$-dToda hierarchy with a finite number of nonzero times

$$
\begin{aligned}
t_{n} & =\bar{t}_{m}=0, \quad n>n_{1}, m>n_{2}, \\
t_{n_{1}} & \neq 0, \bar{t}_{n_{2}} \neq 0 .
\end{aligned}
$$

The first type of systems is characterized by a string equation of the form

$$
\bar{P}(\overline{\mathcal{L}})=P(\mathcal{L})
$$

The corresponding solutions verify reductions of the dToda hierarchy depending on a finite number of unknown functions so that they represent hodograph 
sectors. In Section 3 we show a natural way of deriving hodograph relations in the twistor method and, as an illustration, several solutions of (5) corresponding to cases of the form

$$
\overline{\mathcal{L}}^{ \pm \beta}=\mathcal{L}^{\alpha}
$$

are exhibited.

Section 4 is devoted to the family of systems of string equations

$$
\overline{\mathcal{L}}^{\beta}=\alpha \frac{\mathcal{L}^{\alpha}}{\mathcal{M}}, \quad \frac{1}{\beta} \overline{\mathcal{M}}=\frac{1}{\alpha} \mathcal{M},
$$

introduced by Takasaki [6] for describing the integrable structure of twodimensional quantum gravity. The case $\alpha=\beta=1$ is specially important since the corresponding solutions of the dToda hierarchy describe the genus zero-limit of two-dimensional quantum gravity coupled to $c=1$ matter $[$. Furthermore, it also underlies the integrable structures in the theory of conformal maps [7] and Laplacian growth problems [8]. We obtain solutions of the $\left(n_{1}, n_{2}\right)$-dToda hierarchy satisfying (10) with

$$
n_{1}>\alpha-\beta, \quad n_{2}>\beta-\alpha \text {. }
$$

These solutions are determined by a system of implicit equations which can be conveniently handed with computer aid. For all the cases considered we provide an equation of hodograph type characterizing the function $\bar{u}_{0}$, so that a corresponding solution of the Boyer-Finley equation is obtained.

\section{The twistor method of solution}

In terms of the differential form

$$
\omega:=d \ln p \wedge d s+\sum_{n \geq 1} d \mathcal{B}_{n} \wedge d t_{n}+\sum_{n \geq 1} d \overline{\mathcal{B}}_{n} \wedge d \bar{t}_{n}
$$

the dToda hierarchy can be formulated in the following concise way

$$
\omega=d \ln \mathcal{L} \wedge d \mathcal{M}=d \ln \overline{\mathcal{L}} \wedge d \overline{\mathcal{M}}
$$


From (11) and (12) it follows the existence of two functions $S=S(\mathcal{L}, t, \bar{t}, s)$ and $\bar{S}=\bar{S}(\overline{\mathcal{L}}, t, \bar{t}, s)$ such that

$$
\begin{aligned}
& d S=\mathcal{M} d \ln \mathcal{L}+\ln p d s+\sum_{n \geq 1} \mathcal{B}_{n} d t_{n}+\sum_{n \geq 1} \overline{\mathcal{B}}_{n} d \bar{t}_{n}, \\
& d \bar{S}=\overline{\mathcal{M}} d \ln \overline{\mathcal{L}}+\ln p d s+\sum_{n \geq 1} \mathcal{B}_{n} d t_{n}+\sum_{n \geq 1} \overline{\mathcal{B}}_{n} d \bar{t}_{n},
\end{aligned}
$$

so that they can be assumed to admit expansions of the form

$$
\begin{aligned}
& S=\sum_{n \geq 1} t_{n} \mathcal{L}^{n}+s \ln \mathcal{L}-\sum_{n \geq 1} \frac{v_{n}(t, \bar{t}, s)}{n} \mathcal{L}^{-n}, \\
& \bar{S}=\sum_{n \geq 1} \bar{t}_{n} \overline{\mathcal{L}}^{-n}+s \ln \overline{\mathcal{L}}+\Phi(x, y, s)+\sum_{n \geq 1} \frac{\bar{v}_{n}(t, \bar{t}, s)}{n} \overline{\mathcal{L}}^{n} .
\end{aligned}
$$

The twistor method for solving the dToda hierarchy is supplied by the following result [3]

Theorem Let $(P(p, s), X(p, s))$ and $(\bar{P}(p, s), \bar{X}(p, s))$ be a pair of canonically conjugate variables (i.e. $\{P, X\}=P,\{\bar{P}, \bar{X}\}=\bar{P})$. If $(\mathcal{L}, \mathcal{M}, \overline{\mathcal{L}}, \overline{\mathcal{M}})$ are functions of $(p, t, \bar{t}, s)$ which admit expansions of the form (11) and (7), and satisfy the equations

$$
P(\mathcal{L}, \mathcal{M})=\bar{P}(\overline{\mathcal{L}}, \overline{\mathcal{M}}), \quad X(\mathcal{L}, \mathcal{M})=\bar{X}(\overline{\mathcal{L}}, \overline{\mathcal{M}}),
$$

then $(\mathcal{L}, \mathcal{M}, \overline{\mathcal{L}}, \overline{\mathcal{M}})$ is a solution of the dToda hierarchy.

At this point two remarks are in order

1. The string equations (15) are meaningful only when they are interpreted as a suitable Riemann-Hilbert problem on the complex plane of the variable $p$. Indeed, $(\mathcal{L}, \mathcal{M})$ must be analytic functions in a neighborhood $D=\{|p|>r\}$ of $p=\infty$ and $(\overline{\mathcal{L}}, \overline{\mathcal{M}})$ must be analytic functions in a neighborhood $\bar{D}=\{|p|<\bar{r}\}$ of $p=0$. Thus the statement of the Theorem holds provided the string equations are satisfied on a common annulus $A \subset D \bigcap \bar{D}$. The proof (see [3]) consists on differentiating (15) with respect to $(p, t, \bar{t}, s)(p \in A)$, then using the Laurent series in $D$ 
and $\bar{D}$ to obtain two expansions of the results in powers of $p$ on $A$ and, finally, identifying coefficients of both expansions.

In the cases considered below we impose conditions for

$$
P(\mathcal{L}, \mathcal{M}), \bar{P}(\overline{\mathcal{L}}, \overline{\mathcal{M}}), X(\mathcal{L}, \mathcal{M}), \bar{X}(\overline{\mathcal{L}}, \overline{\mathcal{M}}),
$$

to be analytic functions of $p$ on $A:=\mathbb{C}-\{0\}$. These conditions play an essential role in our method as they constitute the relations describing the hodograph sectors represented by our first class of solutions and are part of the conditions required to characterize the solutions of Takasaki string equations.

2. It is helpful to use canonical generating functions [20, 21] to introduce pairs of conjugate variables . For example, the condition of canonicity for a pair $(P, X)$

$$
p d P \wedge d X=P d p \wedge d s,
$$

is ensured by defining $(P, X)$ through generating functions $J_{0}=J_{0}\left(P, s_{1}\right)$ or $J_{1}=J_{1}(P, p)$ verifying

$$
d J_{0}=\frac{X}{P} d P+p d s_{1}, \quad d J_{1}=\frac{X}{P} d P-s_{1} d p,
$$

where

$$
s_{1}:=\frac{s}{p}
$$

Equivalently

$$
\begin{aligned}
& p=\frac{\partial J_{0}}{\partial s_{1}}, \quad X=P \frac{\partial J_{0}}{\partial P} \\
& s=-p \frac{\partial J_{1}}{\partial p}, \quad X=P \frac{\partial J_{1}}{\partial P} .
\end{aligned}
$$

\section{$3 \quad$ Hodograph Sectors}

\subsection{Hodograph relations in the twistor formalism}

From generating functions of the form

$$
J_{0}\left(P, s_{1}\right)=f(P) s_{1}+g(P), \quad \bar{J}_{0}\left(\bar{P}, s_{1}\right)=\bar{f}(\bar{P}) s_{1},
$$


we determine two pairs of conjugate variables $(P, X)$ and $(\bar{P}, \bar{X})$ given by

$$
\begin{aligned}
& P=P(p), \quad X=s \frac{\mathrm{d} \ln p}{\mathrm{~d} \ln P}+\frac{\mathrm{d} g(P)}{\mathrm{d} \ln P}, \\
& \bar{P}=\bar{P}(p), \quad \bar{X}=s \frac{\mathrm{d} \ln p}{\mathrm{~d} \ln \bar{P}}
\end{aligned}
$$

where $P=P(p)$ and $\bar{P}=\bar{P}(p)$ are the inverse functions of $f=f(P)$ $\bar{f}=\bar{f}(P)$, respectively. It follows at once that the corresponding string equations are

$$
\bar{P}(\overline{\mathcal{L}})=P(\mathcal{L})
$$

and

$$
\overline{\mathcal{M}} \frac{\partial \ln \overline{\mathcal{L}}}{\partial \ln \bar{P}(\overline{\mathcal{L}})}=\mathcal{M} \frac{\partial \ln \mathcal{L}}{\partial \ln P(\mathcal{L})}+\frac{\partial g(P(\mathcal{L}))}{\partial \ln P(\mathcal{L})} .
$$

The second string equation can be rewritten as

$$
\overline{\mathcal{M}}=\frac{\partial(S+g(\bar{P}(\overline{\mathcal{L}})))}{\partial \ln \overline{\mathcal{L}}}
$$

or, equivalently, in terms of derivatives with respect to the variable $p$

$$
\overline{\mathcal{M}}=\frac{\partial_{p}(S+g(P(\mathcal{L})))}{\partial_{p} P(\mathcal{L})} \overline{\mathcal{L}} \bar{P}^{\prime}(\overline{\mathcal{L}})
$$

We may design a method of solution of (19) and (22) provided the following conditions are satisfied :

A1) There exists a solution $\mathcal{L}=\mathcal{L}(p, w)$ and $\overline{\mathcal{L}}=\overline{\mathcal{L}}(p, w)$ of (19) , of the form (11), depending on a finite number $N$ of unknown coefficients $w:=$ $\left(w_{1}, \ldots, w_{N}\right)$.

A2) The function $\partial_{p} \ln P(\mathcal{L}(p, w))$ vanishes at exactly $N$ different points $p_{i}=p_{i}(w)$

Indeed, under these assumptions we determine the unknowns $w$ by imposing

$$
S+g(P(\mathcal{L}))=\sum_{n=1}^{n_{1}} t_{n} \mathcal{B}_{n}+\sum_{n=1}^{n_{2}} \bar{t}_{n} \overline{\mathcal{B}}_{n}+s \ln p+(g(P(\mathcal{L})))_{\geq 0}
$$


and the vanishing of the numerator of (22) at the points $p_{i}$. Thus we get the $N$ hodograph relations

$$
\sum_{n=1}^{n_{1}} t_{n} \partial_{p} \mathcal{B}_{n}\left(p_{i}\right)+\sum_{n=1}^{n_{2}} \bar{t}_{n} \partial_{p} \overline{\mathcal{B}}_{n}\left(p_{i}\right)+\frac{s}{p_{i}}+h\left(p_{i}\right)=0
$$

where

$$
h(p):=\partial_{p}(g(P(\mathcal{L}(p, w))))_{\geq 0},
$$

and find $\overline{\mathcal{M}}$ from (22). Notice that the hodograph relations prevent $\overline{\mathcal{M}}$ from having poles at the points $p_{i}$. Moreover, near $p=0$ the form of $\overline{\mathcal{M}}$ satisfies (17) since from (23) and by taking into account the assumption A1) we deduce

$$
S+g(P(\mathcal{L}))=\sum_{n=1}^{n_{2}} \bar{t}_{n} \overline{\mathcal{L}}^{-n}+s \ln \overline{\mathcal{L}}+\mathcal{O}(1), \quad \overline{\mathcal{L}} \rightarrow 0
$$

and therefore

$$
\overline{\mathcal{M}}=\frac{\partial(S+g(P(\mathcal{L})))}{\partial \ln \overline{\mathcal{L}}}=-\sum_{n=1}^{n_{2}} n \bar{t}_{n} \overline{\mathcal{L}}^{-n}+s+\mathcal{O}(\overline{\mathcal{L}})
$$

Similarly, the expression (23) for $S$ leads to a function $\mathcal{M}$ with an expansion of the form (17).

We notice that for the simplest case $n_{1}=n_{2}=1$, (24) becomes

$$
x+\frac{s}{p_{i}}-\frac{\bar{u}_{0}(w)}{p_{i}^{2}} y+h\left(p_{i}\right)=0,
$$

which coincides with the system of hodograph relations for the Boyer-Finley equation found in 15 .

\subsection{Examples}

If we take

$$
\overline{\mathcal{L}}^{\beta}=\mathcal{L}^{\alpha}, \quad \alpha>\beta>0,
$$

then it is immediate to see that expressions of the form

$$
\begin{aligned}
& \overline{\mathcal{L}}=\left(p^{\beta} w_{1}+\cdots+p^{\alpha-1} w_{\alpha-\beta}+p^{\alpha}\right)^{\frac{1}{\beta}}, \\
& \mathcal{L}=\left(p^{\beta} w_{1}+\cdots+p^{\alpha-1} w_{\alpha-\beta}+p^{\alpha}\right)^{\frac{1}{\alpha}}
\end{aligned}
$$


solve (25), depend on the $\alpha-\beta$ unknown coefficients $w=\left(w_{1}, \ldots, w_{\alpha-\beta}\right)$ and have expansions of the form (11). Furthermore,

$$
\partial_{p} \ln P(\mathcal{L})=\frac{\alpha p^{\alpha-\beta}+(\alpha-1) p^{\alpha-\beta-1} w_{\alpha-\beta}+\cdots+\beta w_{1}}{p^{\alpha-\beta+1}+\cdots+p w_{1}},
$$

has exactly $\alpha-\beta$ zeros $p_{i}=p_{i}(w)$. Hence, the assumptions A1) and A2) are satisfied and the hodograph relations (24) determine solutions of the truncated dToda hierarchy.

For example if

$$
\alpha=2, \quad \beta=1, \quad g(P)=0,
$$

we have $w=w_{1}, \bar{u}_{0}=1 / w_{1}$ and it follows an hodograph relation for the $(1,1)$-dToda hierarchy of the form

$$
x-4 y \bar{u}_{0}^{3}-2 s \bar{u}_{0}=0,
$$

which provides the solution

$$
\begin{aligned}
\bar{u}_{0} & =\frac{s}{3^{\frac{1}{3}}} \sqrt{y}\left(-9 x \sqrt{y}+\sqrt{3} \sqrt{8 s^{3}+27 x^{2} y}\right)^{-\frac{1}{3}} \\
& -\frac{1}{2 \cdot 3^{\frac{2}{3}} \sqrt{y}}\left(-9 x \sqrt{y}+\sqrt{3} \sqrt{8 s^{3}+27 x^{2} y}\right)^{\frac{1}{3}}
\end{aligned}
$$

Other solutions of the $(1,1)$-dToda hierarchy are

1) $\alpha=3, \quad \beta=1, \quad g(P)=0$

$$
w=\left(w_{1}, w_{2}\right), \quad \bar{u}_{0}=\frac{1}{w_{1}} .
$$

The system of hodograph equations for $w$ is

$$
\begin{aligned}
& x-\frac{6 s}{2 w_{2}+\sqrt{-12 w_{1}+4 w_{2}^{2}}}-\frac{36 y}{w_{1}\left(-2 w_{2}-\sqrt{-12 w_{1}+4 w_{2}^{2}}\right)^{2}}=0 \\
& x-\frac{6 s}{2 w_{2}-\sqrt{-12 w_{1}+4 w_{2}^{2}}}-\frac{36 y}{w_{1}\left(-2 w_{2}+\sqrt{-12 w_{1}+4 w_{2}^{2}}\right)^{2}}=0
\end{aligned}
$$

which leads to the following implicit equation for $\bar{u}_{0}$

$$
\left(x+3 y \bar{u}_{0}^{2}\right)\left(-3 s^{2} \bar{u}_{0}+\left(x-3 y \bar{u}_{0}^{2}\right)^{2}\right)=0 .
$$


2) $\alpha=3, \quad \beta=2, \quad g(P)=0$

$$
w=w_{1}, \quad \bar{u}_{0}=\frac{1}{w_{1}^{1 / 2}} .
$$

We get the hodograph equation

$$
-6 s \bar{u}_{0}^{2}+4 x-9 y \bar{u}_{0}^{5}=0 .
$$

3) $\alpha=2, \quad \beta=1, \quad g(P)=P^{\frac{3}{2}}$

$$
w=w_{1}, \quad \bar{u}_{0}=\frac{1}{w_{1}} .
$$

The hodograph equation is

$$
-3-16 s \bar{u}_{0}^{3}+8 x \bar{u}_{0}^{2}-32 y \bar{u}_{0}^{5}=0 .
$$

For the choice

$$
\frac{1}{\overline{\mathcal{L}}^{\beta}}=\mathcal{L}^{\alpha}, \quad \alpha, \beta>0
$$

it follows that

$$
\begin{aligned}
\frac{1}{\overline{\mathcal{L}}} & =\left(\frac{w_{-\beta}}{p^{\beta}}+\cdots+\frac{w_{-1}}{p}+w_{1}+p w_{2}+\cdots+p^{\alpha-1} w_{\alpha}+p^{\alpha}\right)^{\frac{1}{\beta}}, \\
\mathcal{L} & =\left(\frac{w_{-\beta}}{p^{\beta}}+\cdots+\frac{w_{-1}}{p}+w_{1}+p w_{2}+\cdots+p^{\alpha-1} w_{\alpha}+p^{\alpha}\right)^{\frac{1}{\alpha}},
\end{aligned}
$$

solve (30), depend on the $\alpha+\beta$ unknown coefficients $w=\left(w_{-\beta}, \ldots, w_{-1}, w_{1}, \ldots, w_{\alpha}\right)$ and have expansions of the form (1). Moreover,

$$
\partial_{p} \ln P(\mathcal{L})=\frac{\alpha p^{\alpha+\beta}+(\alpha-1) p^{\alpha+\beta-1} w_{\alpha}+\cdots-\beta w_{-\beta}}{p^{\alpha+\beta+1}+\cdots+p w_{-\beta}},
$$

has exactly $\alpha+\beta$ zeros $p_{i}=p_{i}(w)$. Hence, the assumptions A1) and A2) are satisfied so that the hodograph relations (24) determine solutions of the dToda hierarchy.

For example if $\alpha=2, \quad \beta=1, \quad g(P)=P^{-3}$, then

$$
w=\left(w_{-1}, w_{1}, w_{2}\right), \quad \bar{u}_{0}=w_{-1} .
$$


In this case the hodograph equations for $w$ lead to the system

$$
\begin{gathered}
12 w_{1} w_{-1}+x=0, \\
s+6 w_{1} w_{2} w_{-1}+6 w_{-1}^{2}=0, \\
3 w_{1}^{2} w_{-1}+6 w_{2} w_{-1}^{2}-y w_{-1}=0,
\end{gathered}
$$

which implies the following implicit relation for $\bar{u}_{0}$

$$
-x^{3}+48 x y \bar{u}_{0}^{2}-576 s \bar{u}_{0}^{3}-3456 \bar{u}_{0}^{5}=0 .
$$

\section{Takasaki String Equations}

\subsection{General scheme}

By taking the generating functions

$$
J_{1}(P, p)=-\frac{p^{\alpha}}{P}, \quad \bar{J}_{0}\left(\bar{P}, s_{1}\right)=\bar{P}^{1 / \beta} s_{1}, \quad \alpha, \beta>0,
$$

we determine the pairs of conjugate variables

$$
\begin{aligned}
& P=\alpha \frac{p^{\alpha}}{s}, \quad X=\frac{s}{\alpha}, \\
& \bar{P}=p^{\beta}, \quad \bar{X}=\frac{s}{\beta} .
\end{aligned}
$$

They lead to the string equations proposed by Takasaki

$$
\overline{\mathcal{L}}^{\beta}=\alpha \frac{\mathcal{L}^{\alpha}}{\mathcal{M}}, \quad \frac{1}{\beta} \overline{\mathcal{M}}=\frac{1}{\alpha} \mathcal{M},
$$

or equivalently

$$
\begin{aligned}
& \beta \mathcal{M}=\alpha \overline{\mathcal{M}}, \\
& \beta \frac{\mathcal{L}^{\alpha}}{\overline{\mathcal{L}}^{\beta}}=\overline{\mathcal{M}} .
\end{aligned}
$$


We next prove that the string equations (33), (34) have solutions satisfying (11) and (7) with

$$
\begin{aligned}
& \mathcal{M}=\sum_{n=1}^{n_{1}} n t_{n} \mathcal{L}^{n}+s+\sum_{n=1}^{\infty} v_{n} \mathcal{L}^{-n}, \\
& \overline{\mathcal{M}}=-\sum_{n=1}^{n_{2}} n \bar{t}_{n} \overline{\mathcal{L}}^{-n}+s+\sum_{n=1}^{\infty} \bar{v}_{n} \overline{\mathcal{L}}^{n} .
\end{aligned}
$$

Given two integers $r \leq s$ let us denote by $\mathrm{V}[r, s]$ the set of truncated Laurent series of the form

$$
c_{r} p^{r}+c_{r+1} p^{r+1}+\cdots+c_{s} p^{s} .
$$

Let us look for solutions of (33)-(34) such that $\mathcal{L}^{\alpha}$ and $\overline{\mathcal{L}}^{-\beta}$ are meromorphic functions of $p$ with possible poles only at $p=0$ and $p=\infty$. Then, as a consequence of the assumptions (11), (35) and the twistor equations

$$
\frac{\alpha}{\overline{\mathcal{L}}^{\beta}}=\mathcal{M} \mathcal{L}^{-\alpha}, \quad \beta \mathcal{L}^{\alpha}=\overline{\mathcal{M}} \overline{\mathcal{L}}^{\beta}
$$

it follows that

$$
\frac{1}{\overline{\mathcal{L}}^{\beta}} \in \mathrm{V}\left[-\beta, n_{1}-\alpha\right], \quad \mathcal{L}^{\alpha} \in \mathrm{V}\left[\beta-n_{2}, \alpha\right]
$$

Thus, the existence of nontrivial solutions requieres

$$
n_{1}>\alpha-\beta, \quad n_{2}>\beta-\alpha .
$$

We can split (33) into the system of equations:

$$
\begin{gathered}
\alpha \overline{\mathcal{M}}_{\geq 1}=\beta \mathcal{M}_{\geq 1}, \\
\alpha \overline{\mathcal{M}}_{0}=\beta \mathcal{M}_{0}, \\
\alpha \overline{\mathcal{M}}_{\leq-1}=\beta \mathcal{M}_{\leq-1},
\end{gathered}
$$


where $(\ldots)_{\geq 1}\left((\ldots)_{\leq-1}\right)$ denote the part of the Laurent series with strictly positive (strictly negative) powers of $p$, and (...) $)_{0}$ stands for the constant term. Obviously this system is satisfied if we set

$$
\begin{aligned}
& \mathcal{M}=\sum_{n=1}^{n_{1}} n t_{n}\left(\mathcal{L}^{n}\right)_{\geq 1}+\frac{\alpha}{\beta} \overline{\mathcal{M}}_{0}-\frac{\alpha}{\beta} \sum_{n=1}^{n_{2}} n \bar{t}_{n}\left(\overline{\mathcal{L}}^{-n}\right)_{\leq-1}, \\
& \overline{\mathcal{M}}=-\sum_{n=1}^{n_{2}} n \bar{t}_{n}\left(\overline{\mathcal{L}}^{-n}\right)_{\leq-1}+\overline{\mathcal{M}}_{0}+\frac{\beta}{\alpha} \sum_{n=1}^{n_{1}} n t_{n}\left(\mathcal{L}^{n}\right)_{\geq 1},
\end{aligned}
$$

where

$$
\overline{\mathcal{M}}_{0}=s-\sum_{n=1}^{n_{2}} n \bar{t}_{n}\left(\overline{\mathcal{L}}^{-n}\right)_{0} .
$$

Moreover, from (41) it can be easily seen that $\overline{\mathcal{M}}$ has the required expansion of the form (17) provided $\mathcal{L}$ and $\overline{\mathcal{L}}$ satisfy (11). On the other hand, the expression (41) for $\mathcal{M}$ has an expansion of the form (35) if the residue of $\mathcal{M ~}^{-1}$ corresponding to its Laurent expansion in powers of $\mathcal{L}$ verifies

$$
\operatorname{Res}\left(\frac{\mathcal{M}}{\mathcal{L}}, \mathcal{L}\right)=s
$$

Hence the problem reduces to finding $\mathcal{L}$ and $\overline{\mathcal{L}}$ satisfying (11), (34) and (42).

In view of (11) and (36) we look for $\overline{\mathcal{L}}^{-\beta}$ and $\mathcal{L}^{\alpha}$ in the form

$$
\begin{aligned}
& \overline{\mathcal{L}}^{-\beta}=\frac{\bar{w}_{0}}{p^{\beta}}+\frac{\bar{w}_{1}}{p^{\beta-1}}+\cdots+\frac{\bar{w}_{m}}{p^{\beta-m}}, \quad m:=n_{1}-\alpha+\beta, \\
& \mathcal{L}^{\alpha}=p^{\alpha}+w_{1} p^{\alpha-1}+\cdots+w_{\alpha-\beta+n_{2}} p^{\beta-n_{2}} .
\end{aligned}
$$

Hence (34) reads

$$
\beta \mathcal{L}^{\alpha}=\frac{p^{\beta} \overline{\mathcal{M}}}{\bar{w}_{m} p^{m}+\cdots+\bar{w}_{1} p+\bar{w}_{0}},
$$

and in order to prevent $\mathcal{L}^{\alpha}$ from having poles different from $p=0$ and $p=\infty$ we impose

$$
\overline{\mathcal{M}}\left(p_{i}(\bar{w})\right)=0
$$


where $p_{i}(\bar{w}),\left(\bar{w}:=\left(\bar{w}_{1}, \cdots, \bar{w}_{m}\right)\right)$ denote the $m$ zeros of

$$
\bar{w}_{m} p^{m}+\cdots+\bar{w}_{1} p+\bar{w}_{0}=0 .
$$

In this way by using (43) in the expression (41) for $\overline{\mathcal{M}}$, the equation (44) becomes dependent on the variables

$$
\left(p, s, t, \bar{t}, \bar{w}_{0}, \ldots, \bar{w}_{m}, w_{1}, \ldots, w_{\alpha-\beta+n_{2}}\right)
$$

Thus, by identifying coefficients of the powers $p^{i}, i=\beta-n_{2}, \ldots, \alpha$ we get $\alpha-\beta+n_{2}+1$ equations which together with the $m$ equations (45) determine the $\alpha-\beta+n_{2}+m+1$ unknowns variables $\left(\bar{w}_{0}, \ldots, \bar{w}_{m}, w_{1}, \ldots, w_{\alpha-\beta+n_{2}}\right)$ as functions of $(s, t, \bar{t})$. However, to complete our proof we must show that (42) is satisfied too. To do that let us take two circles $\gamma(|p|=r)$ and $\bar{\gamma}(|p|=\bar{r})$ in the complex $p$-plane and denote by $\Gamma$ and $\bar{\Gamma}$ their images under the maps $\mathcal{L}=\mathcal{L}(p)$ and $\overline{\mathcal{L}}=\overline{\mathcal{L}}(p)$, respectively. Then we have

$$
\begin{aligned}
& \operatorname{Res}\left(\frac{\mathcal{M}}{\mathcal{L}}, \mathcal{L}\right)-\operatorname{Res}\left(\frac{\overline{\mathcal{M}}}{\overline{\mathcal{L}}}, \overline{\mathcal{L}}\right) \\
& =\frac{1}{2 i \pi} \oint_{\Gamma} \frac{\mathcal{M}}{\mathcal{L}} \mathrm{d} \mathcal{L}-\frac{1}{2 i \pi} \oint_{\bar{\Gamma}} \frac{\overline{\mathcal{M}}}{\overline{\mathcal{L}}} \mathrm{d} \overline{\mathcal{L}} \\
& =\frac{1}{2 i \pi} \oint_{\gamma} \frac{\partial_{p} \mathcal{L}^{\alpha}}{\overline{\mathcal{L}}^{\beta}} \mathrm{d} p+\frac{1}{2 i \pi} \oint_{\bar{\gamma}} \mathcal{L}^{\alpha} \partial_{p}\left(\overline{\mathcal{L}}^{-\beta}\right) \mathrm{d} p \\
& =\frac{1}{2 i \pi} \oint_{\gamma} \partial_{p}\left(\frac{\mathcal{L}^{\alpha}}{\overline{\mathcal{L}}^{\beta}}\right) \mathrm{d} p=0,
\end{aligned}
$$

where we have taken into account that the integrands are analytic functions of $p$ in $\mathbb{C}-\{0\}$ and that $\gamma$ and $\bar{\gamma}$ are homotopic with respect to $\mathbb{C}-\{0\}$. Therefore, as we have already proved that $\overline{\mathcal{M}}$ has an expansion of the form (17), we deduce

$$
\operatorname{Res}\left(\frac{\mathcal{M}}{\mathcal{L}}, \mathcal{L}\right)=\operatorname{Res}\left(\frac{\overline{\mathcal{M}}}{\overline{\mathcal{L}}}, \overline{\mathcal{L}}\right)=s
$$

so that (42) follows. 


\subsection{Examples}

We first illustrate our method by considering two cases with $\alpha=\beta$.

I) $\alpha=\beta, n_{1}=n_{2}=1$

The starting point is to set

$$
\begin{aligned}
& \mathcal{L}^{\alpha}=p^{\alpha}+w_{1} p^{\alpha-1}, \\
& \overline{\mathcal{L}}^{-\alpha}=\frac{\bar{w}_{0}}{p^{\alpha}}+\frac{\bar{w}_{1}}{p^{\alpha-1}} .
\end{aligned}
$$

The polynomial $p^{\alpha} \overline{\mathcal{L}}^{-\alpha}$ has a unique zero at

$$
p_{1}=-\frac{\bar{w}_{0}}{\bar{w}_{1}}
$$

thus, (46) leads us to

$$
s-x \frac{\bar{w}_{0}}{\bar{w}_{1}}+\left(1-\frac{1}{\alpha}\right) y \bar{w}_{0}^{-1+\frac{1}{\alpha}} \bar{w}_{1}=0
$$

Now, by equating the powers of $p$ in (45) we obtain

$$
\begin{aligned}
p^{\alpha}: & \alpha \bar{w}_{1}+x=0 \\
p^{\alpha-1}: & \alpha w_{1}-\frac{s}{\bar{w}_{1}}+\frac{x \bar{w}_{0}}{\bar{w}_{1}^{2}}+\frac{y \bar{w}_{0}^{-1+\frac{1}{\alpha}}}{\alpha}=0
\end{aligned}
$$

Finally, from (46) and (47) we can eliminate $\bar{w}_{1}$ and $w_{1}$, and taking into account that $\bar{w}_{0}=\bar{u}_{0}^{\alpha}$ we get $\bar{u}_{0}$ implicitly defined by

$$
(1-\alpha) x y \bar{u}_{0}-\alpha^{2} s \bar{u}_{0}^{\alpha}+\alpha^{3} \bar{u}_{0}^{2 \alpha}=0 .
$$

II) $\alpha=\beta, n_{1}=2, n_{2}=1$

We start with the expressions

$$
\begin{gathered}
\mathcal{L}^{\alpha}=p^{\alpha}+w_{1} p^{\alpha-1} \\
\overline{\mathcal{L}}^{-\alpha}=\frac{\bar{w}_{0}}{p^{\alpha}}+\frac{\bar{w}_{1}}{p^{\alpha-1}}+\frac{\bar{w}_{2}}{p^{\alpha-2}}
\end{gathered}
$$


Now the polynomial $p^{\alpha} \overline{\mathcal{L}}^{-\alpha}$ has two zeros at the points

$$
p_{1}=\frac{-\bar{w}_{1}+\sqrt{\bar{w}_{1}^{2}-4 \bar{w}_{0} \bar{w}_{2}}}{2 \bar{w}_{2}}, \quad p_{2}=\frac{-\bar{w}_{1}-\sqrt{\bar{w}_{1}^{2}-4 \bar{w}_{0} \bar{w}_{2}}}{2 \bar{w}_{2}},
$$

thus, (46) yields two equations which become equivalent to

$$
\begin{gathered}
2 \alpha t_{2} \bar{w}_{0} \bar{w}_{1}^{2}-2 \alpha t_{2} \bar{w}_{0}^{2} \bar{w}_{2}-4 t_{2} \bar{w}_{0} w_{1} \bar{w}_{1} \bar{w}_{2}+\alpha s \bar{w}_{0} \bar{w}_{2}^{2} \\
-\alpha x \bar{w}_{0} \bar{w}_{1} \bar{w}_{2}-y \bar{w}_{0}^{\frac{1}{\alpha}} \bar{w}_{1} \bar{w}_{2}^{2}=0, \\
2 \alpha t_{2} \bar{w}_{0}^{2} \bar{w}_{1}-4 t_{2} \bar{w}_{0}^{2} w_{1} \bar{w}_{2}-\alpha x \bar{w}_{0}^{2} \bar{w}_{2}-\alpha y \bar{w}_{0}^{1+\frac{1}{\alpha}} \bar{w}_{2}^{2}=0 .
\end{gathered}
$$

Now, by identifying coefficients of powers of $p$ in (45) we obtain

$$
\begin{aligned}
p^{\alpha}: & -2 t_{2}+\alpha \bar{w}_{2}=0 \\
p^{\alpha-1}: & 2 a t_{2} \bar{w}_{1}-4 t_{2} w_{1} \bar{w}_{2}+\alpha^{2} w_{1} \bar{w}_{2}^{2}-\alpha x \bar{w}_{2}=0
\end{aligned}
$$

Finally, from (49), (50) and (51) we can eliminate $\bar{w}_{1}, \bar{w}_{2}$ and $w_{1}$, and taking into account that $\bar{w}_{0}=\bar{u}_{0}^{\alpha}$ we get the following implicit equation for $\bar{u}_{0}$

$$
\begin{aligned}
& \alpha^{4} s \bar{u}_{0}^{2 \alpha}-\alpha^{5} \bar{u}_{0}^{3 \alpha}+\alpha^{3} x y \bar{u}_{0}^{\alpha+1}+4 t_{2} y^{2} \bar{u}_{0}^{2}-6 \alpha t_{2} y^{2} \bar{u}_{0}^{2} \\
& +\alpha^{2} y \bar{u}_{0}\left(-x \bar{u}_{0}^{\alpha}+2 t_{2} y \bar{u}_{0}\right)=0 .
\end{aligned}
$$

Next we quote the final implicit relation for $\bar{u}_{0}$ corresponding to some examples of solutions for $\alpha \neq \beta$.

1) $\alpha=4, \beta=2, n_{1}=3, n_{2}=1$.

$$
2560 \bar{u}_{0}^{7}-1536 t_{2} \bar{u}_{0}^{5}+64\left(2 t_{2}^{2}+3 t_{3} x\right) \bar{u}_{0}^{3}-72 t_{3}^{2} s \bar{u}_{0}-27 t_{3}^{3} y=0 .
$$

2) $\alpha=3, \beta=2, n_{1}=2, n_{2}=1$.

$$
3 s t_{2} \bar{u}_{0}+27 \bar{u}_{0}^{5}-9 x \bar{u}_{0}^{3}+t_{2}^{2} y=0 .
$$


3) $\alpha=5, \beta=3, n_{1}=3, n_{2}=1$

$$
\begin{gathered}
-120 t_{3}^{2} s \bar{u}_{0}^{2}+100 t_{2}{ }^{2} \bar{u}_{0}^{5}-2000 t_{2} \bar{u}_{0}^{8}+4375 \bar{u}_{0}^{11} \\
+300 t_{3} x \bar{u}_{0}^{5}-48 t_{3}^{3} y=0 .
\end{gathered}
$$

4) $\alpha=2, \beta=1, n_{1}=3, n_{2}=1$.

$$
-3 t_{3} s^{2}-16 \bar{u}_{0}^{3}+8 x \bar{u}_{0}^{2}-12 t_{3} \text { y } \bar{u}_{0}^{2}=0 .
$$

5) $\alpha=3, \beta=1, n_{1}=4, n_{2}=1$

$$
\begin{gathered}
-64 t_{4}{ }^{3} s^{3}+288 t_{2} t_{4}{ }^{2} s^{2} \bar{u}_{0}-1296 t_{4}{ }^{2} s^{2} \bar{u}_{0}^{2}-864 t_{2}{ }^{3} \bar{u}_{0}^{3} \\
+1296 t_{2} t_{4} s \bar{u}_{0}^{3}+7776 t_{2}{ }^{2} \bar{u}_{0}^{4}-4860 t_{4} s \bar{u}_{0}^{4}-21870 t_{2} \bar{u}_{0}^{5} \\
+18225 \bar{u}_{0}^{6}+972 t_{4} x^{2} \bar{u}_{0}^{3}-2592 t_{4}{ }^{2} x y \bar{u}_{0}^{3}+1728 t_{4}{ }^{3} y^{2} \bar{u}_{0}^{3}=0 .
\end{gathered}
$$

\subsection{Applications to integrable contour dynamics}

Let $z=z(p)$ be an invertible conformal map of the exterior of the unit circle $|p|>1$ to the exterior of a simply connected domain bounded by a simple analytic curve $\gamma$. At $p \rightarrow \infty$ it can be expanded in the form

$$
z(p)=r p+\sum_{n=0}^{\infty} \frac{r_{n}}{p^{n}},
$$

where the coefficient $r$ is real. By expressing the coefficients $\left(r, r_{0}, r_{1}, \ldots\right)$ as functions of the harmonic moments $t=\left(t_{0}, t_{1}, \ldots\right)$ of the exterior of $\gamma$ it turns out [7]-[8] that the corresponding function $z(p, t)$ determines a solution of the dToda hierarchy. The relation between the dynamical objects involved in the two different gauges of the dToda hierarchy used in [1]-[3] and [6]-[7] is as follows

$$
z(p)=\mathcal{L}(r p), \quad \bar{z}\left(\frac{1}{p}\right)=\overline{\mathcal{L}}^{-1}(r p), \quad r:=\sqrt{\bar{u}_{0}},
$$

where

$$
\bar{z}\left(\frac{1}{p}\right)=\frac{r}{p}+\sum_{n=0}^{\infty} \frac{r_{n}^{*}}{p^{n}},
$$


and

$$
t_{0}=s, \quad \bar{t}_{n}=-t_{n}^{*}, \quad n \geq 1 .
$$

The associated system of string equations is

$$
\overline{\mathcal{L}}=\frac{\mathcal{L}}{\mathcal{M}}, \quad \overline{\mathcal{M}}=\mathcal{M},
$$

which implies

$$
\left\{z(p), \bar{z}\left(\frac{1}{p}\right)\right\}=1 .
$$

Furthermore, in view of (154)-(155), the solution satisfies the reduction condition

$$
\frac{1}{\overline{\mathcal{L}}(r p)}=(\mathcal{L}(r p))^{*}, \quad \text { for }|p|=1 .
$$

It can be seen that the method developed in Subsection 4.1 is compatible with (58) provided $r$ is real, so that it can be applied to obtain these solutions by setting

$$
\alpha=\beta=1, \quad n_{1}=n_{2} .
$$

The following two examples illustrate the simplest cases.

I) $n_{1}=n_{2}=2$ (the ellipse)

The polynomial $p \overline{\mathcal{L}}^{-1}$ has two zeros at the points

$$
p_{1}=\frac{-\bar{w}_{1}+\sqrt{\bar{w}_{1}^{2}-4 \bar{w}_{0} \bar{w}_{2}}}{2 \bar{w}_{2}}, \quad p_{2}=\frac{-\bar{w}_{1}-\sqrt{\bar{w}_{1}^{2}-4 \bar{w}_{0} \bar{w}_{2}}}{2 \bar{w}_{2}} .
$$

From (45) we get two equations which lead to

$$
\begin{aligned}
& -2 t_{2} \bar{w}_{1}^{3}+4 t_{2} \bar{w}_{0} \bar{w}_{1} \bar{w}_{2}+t_{1} \bar{w}_{1}^{2} \bar{w}_{2}+4 t_{2} w_{1} \bar{w}_{1}^{2} \bar{w}_{2}-t_{1} \bar{w}_{0} \bar{w}_{2}^{2}-4 t_{2} \bar{w}_{0} w_{1} \bar{w}_{2}^{2} \\
& -t_{0} \bar{w}_{1} \bar{w}_{2}^{2}-t_{1}^{*} \bar{w}_{1}^{2} \bar{w}_{2}^{2}-2 t_{2}^{*} \bar{w}_{1}^{3} \bar{w}_{2}^{2}+t_{1}^{*} \bar{w}_{0} \bar{w}_{2}^{3}=0 \\
& -2 t_{2} \bar{w}_{0} \bar{w}_{1}^{2}+2 t_{2} \bar{w}_{0}^{2} \bar{w}_{2}+t_{1} \bar{w}_{0} \bar{w}_{1} \bar{w}_{2}+4 t_{2} \bar{w}_{0} w_{1} \bar{w}_{1} \bar{w}_{2}-t_{0} \bar{w}_{0} \bar{w}_{2}^{2} \\
& -t_{1}^{*} \bar{w}_{0} \bar{w}_{1} \bar{w}_{2}^{2}-2 t_{2}^{*} \bar{w}_{0} \bar{w}_{1}^{2} \bar{w}_{2}^{2}-2 t_{2}^{*} \bar{w}_{0}^{2} \bar{w}_{2}^{3}=0 .
\end{aligned}
$$


Identification of powers of $p$ in (44) implies

$$
\begin{aligned}
p: & -2 t_{2}+\bar{w}_{2}=0, \\
p^{0}: & 2 t_{2} \bar{w}_{1}-t_{1} \bar{w}_{2}-4 t_{2} w_{1} \bar{w}_{2}+w_{1} \bar{w}_{2}^{2}=0, \\
p^{-1}: \quad & -2 t_{2} \bar{w}_{1}^{2}+2 t_{2} \bar{w}_{0} \bar{w}_{2}+t_{1} \bar{w}_{1} \bar{w}_{2}+4 t_{2} w_{1} \bar{w}_{1} \bar{w}_{2}-t_{0} \bar{w}_{2}^{2}-t_{1}^{*} \bar{w}_{1} \bar{w}_{2}^{2} \\
& -2 t_{2}^{*} \bar{w}_{1}^{2} \bar{w}_{2}^{2}-4 t_{2}^{*} \bar{w}_{0} \bar{w}_{2}^{3}+w_{2} \bar{w}_{2}^{3}=0 .
\end{aligned}
$$

By solving equations (59)-(61) we get the solution:

$$
\begin{gathered}
\mathcal{L}=p+\frac{t_{1}^{*}+2 t_{1} t_{2}^{*}}{1-4 t_{2} t_{2}^{*}}+\frac{2 t_{0} t_{2}^{*}}{p\left(1-4 t_{2} t_{2}^{*}\right)}, \\
\frac{1}{\overline{\mathcal{L}}}=\frac{t_{0}}{p\left(1-4 t_{2} t_{2}^{*}\right)}+\frac{t_{1}+2 t_{1}^{*} t_{2}}{1-4 t_{2} t_{2}^{*}}+2 p t_{2},
\end{gathered}
$$

which leads to the conformal map describing an ellipse growing from a circle 6]

$$
z=\left(\frac{t_{0}}{1-4 t_{2} t_{2}^{*}}\right)^{\frac{1}{2}} p+\frac{t_{1}^{*}+2 t_{1} t_{2}^{*}}{1-4 t_{2} t_{2}^{*}}+2\left(\frac{t_{0}}{1-4 t_{2} t_{2}^{*}}\right)^{\frac{1}{2}} \frac{t_{2}^{*}}{p}
$$

\section{II) $n_{1}=n_{2}=3$ (the hypotrochoid)}

Let us take $t_{1}=t_{2}=t_{1}^{*}=t_{2}^{*}=0$, then $p \overline{\mathcal{L}}^{-1}$ is a third degree polynomial and (45) gives rise to a system of three equations which can be reduced to

$$
\begin{aligned}
& 3 t_{3} \bar{w}_{2}^{4}-9 t_{3} \bar{w}_{1} \bar{w}_{2}^{2} \bar{w}_{3}-9 t_{3} w_{1} \bar{w}_{2}^{3} \bar{w}_{3}+3 t_{3} \bar{w}_{1}^{2} \bar{w}_{3}^{2}+6 t_{3} \bar{w}_{0} \bar{w}_{2} \bar{w}_{3}^{2} \\
& +18 t_{3} w_{1} \bar{w}_{1} \bar{w}_{2} \bar{w}_{3}^{2}+9 t_{3} w_{1}^{2} \bar{w}_{2}^{2} \bar{w}_{3}^{2}+9 t_{3} w_{2} \bar{w}_{2}^{2} \bar{w}_{3}^{2}-9 t_{3} \bar{w}_{0} w_{1} \bar{w}_{3}^{3} \\
& -9 t_{3} w_{1}^{2} \bar{w}_{1} \bar{w}_{3}^{3}-9 t_{3} \bar{w}_{1} w_{2} \bar{w}_{3}^{3}-t_{0} \bar{w}_{2} \bar{w}_{3}^{3}-3 t_{3}^{*} \bar{w}_{1}^{3} \bar{w}_{2} \bar{w}_{3}^{3} \\
& -18 t_{3}^{*} \bar{w}_{0} \bar{w}_{1} \bar{w}_{2}^{2} \bar{w}_{3}^{3}+9 t_{3}^{*} \bar{w}_{0} \bar{w}_{1}^{2} \bar{w}_{3}^{4}=0
\end{aligned}
$$

$3 t_{3} \bar{w}_{1} \bar{w}_{2}^{3}-6 t_{3} \bar{w}_{1}^{2} \bar{w}_{2} \bar{w}_{3}-3 t_{3} \bar{w}_{0} \bar{w}_{2}^{2} \bar{w}_{3}-9 t_{3} w_{1} \bar{w}_{1} \bar{w}_{2}^{2} \bar{w}_{3}+6 t_{3} \bar{w}_{0} \bar{w}_{1} \bar{w}_{3}^{2}$

$+9 t_{3} w_{1} \bar{w}_{1}^{2} \bar{w}_{3}^{2}+9 t_{3} \bar{w}_{0} w_{1} \bar{w}_{2} \bar{w}_{3}^{2}+9 t_{3} w_{1}^{2} \bar{w}_{1} \bar{w}_{2} \bar{w}_{3}^{2}+9 t_{3} \bar{w}_{1} w_{2} \bar{w}_{2} \bar{w}_{3}^{2}$

$-9 t_{3} \bar{w}_{0} w_{1}^{2} \bar{w}_{3}^{3}-t_{0} \bar{w}_{1} \bar{w}_{3}^{3}-3 t_{3}^{*} \bar{w}_{1}^{4} \bar{w}_{3}^{3}-9 t_{3} \bar{w}_{0} w_{2} \bar{w}_{3}^{3}-18 t_{3}^{*} \bar{w}_{0} \bar{w}_{1}^{2} \bar{w}_{2} \bar{w}_{3}^{3}=0$ 


$$
\begin{aligned}
& 3 t_{3} \bar{w}_{0} \bar{w}_{2}^{3}-6 t_{3} \bar{w}_{0} \bar{w}_{1} \bar{w}_{2} \bar{w}_{3}-9 t_{3} \bar{w}_{0} w_{1} \bar{w}_{2}^{2} \bar{w}_{3}+3 t_{3} \bar{w}_{0}^{2} \bar{w}_{3}^{2} \\
& +9 t_{3} \bar{w}_{0} w_{1} \bar{w}_{1} \bar{w}_{3}^{2}+9 t_{3} \bar{w}_{0} w_{1}^{2} \bar{w}_{2} \bar{w}_{3}^{2}+9 t_{3} \bar{w}_{0} w_{2} \bar{w}_{2} \bar{w}_{3}^{2}-t_{0} \bar{w}_{0} \bar{w}_{3}^{3} \\
& -3 t_{3}^{*} \bar{w}_{0} \bar{w}_{1}^{3} \bar{w}_{3}^{3}-18 t_{3}^{*} \bar{w}_{0}^{2} \bar{w}_{1} \bar{w}_{2} \bar{w}_{3}^{3}-6 t_{3}^{*} \bar{w}_{0}^{3} \bar{w}_{3}^{4}=0
\end{aligned}
$$

Now, by equating coefficients of powers of $p$ in (44) we obtain

$$
\begin{aligned}
p: & -3 t_{3}+\bar{w}_{3}=0 \\
p^{0}: \quad & 3 t_{3} \bar{w}_{2}-9 t_{3} w_{1} \bar{w}_{3}+w_{1} \bar{w}_{3}^{2}=0 \\
p^{-1}: \quad & -3 t_{3} \bar{w}_{2}^{2}+3 t_{3} \bar{w}_{1} \bar{w}_{3}+9 t_{3} w_{1} \bar{w}_{2} \bar{w}_{3}-9 t_{3} w_{1}^{2} \bar{w}_{3}^{2} \\
& -9 t_{3} w_{2} \bar{w}_{3}^{2}+w_{2} \bar{w}_{3}^{3}=0 \\
p^{-2}: \quad & 3 t_{3} \bar{w}_{2}^{3}-6 t_{3} \bar{w}_{1} \bar{w}_{2} \bar{w}_{3}-9 t_{3} w_{1} \bar{w}_{2}^{2} \bar{w}_{3}+3 t_{3} \bar{w}_{0} \bar{w}_{3}^{2} \\
& +9 t_{3} w_{1} \bar{w}_{1} \bar{w}_{3}^{2}+9 t_{3} w_{1}^{2} \bar{w}_{2} \bar{w}_{3}^{2}+9 t_{3} w_{2} \bar{w}_{2} \bar{w}_{3}^{2}-t \bar{w}_{3}^{3} \\
& -3 t_{3}^{*} \bar{w}_{1}^{3} \bar{w}_{3}^{3}-18 t_{3}^{*} \bar{w}_{0} \bar{w}_{1} \bar{w}_{2} \bar{w}_{3}^{3}-9 t_{3}^{*} \bar{w}_{0}^{2} \bar{w}_{3}^{4}+w_{3} \bar{w}_{3}^{4}=0
\end{aligned}
$$

Now by setting $w_{1}=\bar{w}_{1}=w_{2}=\bar{w}_{2}=0$ one finds the solution

$$
\mathcal{L}=p+\frac{3 t_{3}^{*} \bar{w}_{0}^{2}}{p^{2}}, \quad \frac{1}{\overline{\mathcal{L}}}=3 p^{2} t_{3}+\frac{\bar{w}_{0}}{p},
$$

with

$$
\bar{w}_{0}=\frac{1-\sqrt{1-72 t_{0} t_{3} t_{3}^{*}}}{36 t_{3} t_{3}^{*}},
$$

which satisfies (58) and leads to the conformal map associated with the hypotrochoid 23]

$$
z=\bar{w}_{0}^{\frac{1}{2}} p+\frac{3 t_{3}^{*} \bar{w}_{0}}{p^{2}}
$$

\section{Acknowledgements}

The authors are grateful to Prof. Manuel Mañas for many useful discussions. 


\section{References}

[1] K. Takasaki and T. Takebe 1991 Lett. Math. Phys. 23205

[2] K. Takasaki and T. Takebe 1993 Lett. Math. Phys. 28165

[3] K. Takasaki and T. Takebe 1995 Rev. Math. Phys. 7743

[4] R. Dijkgraaf, G. Moore and R. Plesser 1993 Nucl. Phys. B394 356

[5] A. Hanany, Y. Oz and M. R. Plesser 1994 Nucl. Phys. B425 150

[6] K. Takasaki 1995 Commun. Math. Phys. 170101

[7] P. W. Wiegmann and P. B. Zabrodin 2000 Comm. Math. Phys. 213523

[8] M. Mineev-Weinstein, P. W. Wiegmann and P. B. Zabrodin 2000 Phys. Rev. Lett. 845106

[9] C. P. Boyer and J. D. Finley 1982 J. Math. Phys. 231126

[10] T. Eguchi, P. B. Gilkey and A. J. Hanson 1980 Phys. Rep. 66213

[11] D. M. J. Calderbank and K. P. Tod 2001 Diff. Geom. Appl. 14199

[12] P. E. Jones and K. P. Tod 1985 Class. Quantum Grav. 2565

[13] R. S. Ward 1990 Class. Quantum Grav. 7 L95

[14] I. M. Krichever 1992 Commun. Pure. Appl. Math. 47437

[15] F. Guil, M. Mañas and L. Martinez Alonso 2003 J. Phys. A: Math. Gen. 364047

[16] M. Mañas and L. Martinez Alonso 2004 Phys. Lett. A 320383

[17] E. V. Ferapontov, D. A. Korotkin and V. A. Shramchenko 2002 Class. Quantum Grav. 19 L205

[18] L. Martina, M. B. Sheftel and P. Winternitz 2001 J. Phys. A: Math. Gen. 349243

[19] M. B. Sheftel 2003 Theor. Math. Phys. 1371743 
[20] K. Takasaki and T. Takebe 1992 Int. J. Mod. Phys. A7 Suppl.1 889

[21] F. Guil, M. Mañas and L. Martinez Alonso 2003 J. Phys. A: Math. Gen. 366457

[22] L. Martinez Alonso and M. Mañas 2003 J. Math. Phys. 443294

[23] R. Teodorescu, E. Bettelheim, O. Agam, A. Zabrodin and P. Wiegmann 2004 Normal random matrix ensemble as a growth problem, arXiv:hep-th/0401165 